\title{
Increased Mortality Among Patients With vs Without Cirrhosis and Autoimmune Hepatitis
}

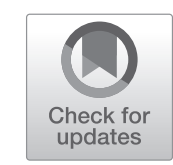

Floris F. van den Brand, ${ }^{*}$ Koen S. van der Veen, ${ }^{*}$ Ynto S. de Boer, ${ }^{*}$ Nicole M. van Gerven, Birgit I. Lissenberg-Witte, ${ }^{\ddagger}$ Ulrich Beuers, ${ }^{\S}$ Karel J. van Erpecum, " Henk R. van Buuren,"

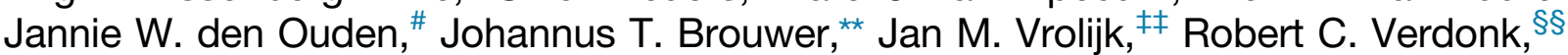
Bart van Hoek, ${ }^{\|\|}$Ger H. Koek, " Chris J. J. Mulder, ${ }^{*}$ Elisabeth Bloemena, ${ }^{\neq \neq \ddagger}$ Carin M. J. van Nieuwkerk, ${ }^{*}$ and Gerd Bouma, ${ }^{*}$ on behalf of the Dutch Autoimmune Hepatitis Study Group

*Amsterdam Gastroenterology and Metabolism, Department of Gastroenterology and Hepatology, Amsterdam UMC, Vrije Universiteit Amsterdam, Amsterdam, the Netherlands; ${ }^{\ddagger}$ Department of Epidemiology and Biostatistics, Amsterdam UMC, Vrije Universiteit Amsterdam, Amsterdam, the Netherlands; ${ }^{\S}$ Department of Gastroenterology and Hepatology, Amsterdam UMC, University of Amsterdam, Amsterdam, the Netherlands; "Department of Gastroenterology and Hepatology, University Medical Center, Utrecht, the Netherlands; "Department of Gastroenterology and Hepatology, Erasmus University Medical Center, Rotterdam, the Netherlands; "Department of Gastroenterology and Hepatology, Haga Hospital, The Hague, the Netherlands; **Department of Gastroenterology and Hepatology, Reinier de Graaf Groep, Delft, the Netherlands; ${ }^{* \pm}$ Department of Gastroenterology and Hepatology, Rijnstate Hospital, Arnhem, the Netherlands; §§Department of Gastroenterology and Hepatology, Sint Antonius Hospital, Nieuwegein, the Netherlands; "Department of Gastroenterology and Hepatology, Leiden University Medical Center, Leiden, the Netherlands; ${ }^{19}$ Department of Internal Medicine, division of Gastroenterology and Hepatology, Maastricht University Medical Center, Maastricht, the Netherlands; "\# Department of Gastroenterology and Hepatology, Radboud University Medical Center, Nijmegen, the Netherlands; ${ }^{* *}$ Department of Gastroenterology and Hepatology, Medisch Spectrum Twente, Enschede, the Netherlands; and ${ }^{\sharp \pm}$ Department of Pathology, Amsterdam UMC, Vrije Universiteit Amsterdam, Amsterdam, the Netherlands

BACKGROUND \& AIMS: $\quad$ There have been few reproducible studies of mortality in patients with autoimmune hepatitis (AIH) and its variants. We calculated mortality in a large national cohort of patients with AIH, with vs without cirrhosis, in the Netherlands.

METHODS:

We collected data from 449 patients with established AIH (77\% female), from 6 academic and 10 non-academic hospitals in the Netherlands. We identified 29 patients with AIH and primary biliary cholangitis and 35 patients with AIH and primary sclerosing cholangitis (AIH-PSC). Mortality and liver transplantation data were assessed from August 1, 2006 through July 31, 2016. Standardized mortality ratios (SMR) were calculated using age-, sex-, and calendar yearmatched mortality for the general Dutch population.

RESULTS:

During the 10-year follow-up period, 60 patients $(13 \%)$ died (mean age, 71 years; range, 33-94 years). Twenty-six causes of death were liver related $(43 \%)$, whereas the others could not be attributed to liver disease. Patients with AIH and cirrhosis had significantly higher mortality than the general population (SMR, 1.9; 95\% CI, 1.2-3.4), whereas patients without cirrhosis did not (SMR, 1.2; 95\% CI, 0.8-1.8). Patients with AIH-PSC had the largest increase in mortality, compared to the general population (SMR, 4.7; 95\% CI, 1.514.6), of all groups analyzed. Mortality in patients with AIH and primary biliary cholangitis was not greater than the general population. Four or more relapses per decade or not achieving remission was associated with an increase in liver-related death or liver transplantation. Nine patients underwent liver transplantation; 2 died from non-liver related causes. Four of 9 patients on the waitlist for transplantation died before receiving a donated liver.

\footnotetext{
Abbreviations used in this paper: AlH, autoimmune hepatitis; AlH-PBC, autoimmune hepatitis with concurrent primary biliary cholangitis; AlHPSC, autoimmune hepatitis with concurrent primary sclerosing cholangitis; ALT, alanine aminotransferase; IAIHG, International Autoimmune Hepatitis Group; SMR, standardized mortality ratio.
}

Most current article

(C) 2019 by the AGA Institute $1542-3565 / \$ 36.00$

https://doi.org/10.1016/j.cgh.2018.09.046 
CONCLUSION:

In an analysis of data from a large national cohort of patients with AIH, we found increased mortality of patients with cirrhosis, but not of patients without cirrhosis, compared to the general Dutch population. Survival was significantly reduced in patients with AIH and features of concurrent PSC.

Keywords: Long Term; Outcome; Autoimmune Liver Disease; Relapse; Remission.

utoimmune hepatitis (AIH) is a chronic immunemediated inflammatory condition. Therapy is aimed to prevent disease progression to fibrosis, cirrhosis, and eventually liver failure and death. Since the introduction of corticosteroid drugs and steroid sparing thiopurine agents, prognosis has improved dramatically. ${ }^{1,2}$ While some studies report that patients with AIH have a good prognosis and normal survival, ${ }^{3-5}$ others contradict these findings. ${ }^{6,7}$ AIH may coexist with primary biliary cholangitis (AIH-PBC) or primary sclerosing cholangitis (AIH-PSC) and recent data suggest that particularly these patients have an impaired outcome. $^{8}$

We previously assessed the incidence and prevalence of AIH in The Netherlands and generated a database of patients derived from general and tertiary referral hospitals. ${ }^{9}$ This cohort has been closely followed. Here, we assessed the mortality and causes of death during 10 years in a clinical cohort of patients with established with vs without cirrhosis and compared this with the general Dutch population.

\section{Materials and Methods}

\section{Patient Population}

Patients with a probable or definite diagnosis of $\mathrm{AIH}$ according to the revised original score of the International Autoimmune Hepatitis Group (IAIHG) ${ }^{10}$ were identified by searching the database of the Dutch Autoimmune Hepatitis Study Group. The study protocol was approved by the institutional review boards of all 6 academic and 10 general hospitals in the Netherlands that participated.

Patients who were alive at the start of the observation period (August 1, 2006), did not have a history of liver transplantation, and had follow-up data available in the medical records were eligible for inclusion. In total, 506 records were reviewed and 449 patients were included. Fifty-seven were excluded for the following reasons: 13 patients were not alive at start of follow-up; 7 patients had undergone liver transplantation before August 1, 2006; and 37 patients did not have follow-up data. In $411(92 \%)$ patients in the study-cohort a liver biopsy was performed, in 38 patients liver biopsy report was not performed or the report could not be retrieved. The diagnosis of AIH was confirmed in all patients using the revised IAIHG score. All patients had a revised, post-treatment IAIHG score of $\geq 12$, confirming the diagnosis of AIH.

Biochemical relapse was defined as an alanine aminotransferase (ALT) $>3$ times upper limit of normal or an increase of the immunoglobulin G level to $>20 \mathrm{~g} / \mathrm{L}^{1}$. Loss of complete biochemical remission was defined as an increase in ALT above the upper limit of normal on 2 occasions with a time interval of at least 4 weeks. ${ }^{11}$ The time to initial remission was defined as the time between diagnosis and biochemical remission. Variant syndrome AIH-PSC was defined as AIH with concurrent PSC based on characteristic liver histology and cholangiographic findings (magnetic resonance cholangiopancreatography and/or endoscopic retrograde cholangiopancreatography). The variant with concurrent PBC was defined as $\mathrm{AIH}$ with an antimitochondrial antibody titer of $>1: 80$ in combination with compatible histology. ${ }^{9}$

Treatment details of immunosuppressive therapy were assessed from the date of diagnosis to the end of follow-up. The cumulative years of medication were registered as the average dose in milligrams per day over 1-year intervals.

\section{Mortality and Liver Transplantation}

Mortality and liver transplantation were assessed during 10 years from August 1, 2006, until July 31, 2016 (end of study). Information on mortality and causes of death were retrieved from medical records and/or by contacting the attending physicians or general practitioners. Liver transplantations were assessed by crosschecking our cohort with data from the 3 liver transplantation centers in the Netherlands. Follow-up ended at the date of death or liver transplantation, or at the end of the study. The cumulative person-years at risk was 4214 years, with a mean of 9.4 years/patient.

\section{Prognostic Factors}

Baseline and follow-up data regarding the age at the start of follow-up, cirrhosis, time to initial remission, amount of relapses and loss of remission, immunoglobulin G and ALT at diagnosis, variant syndromes, and medication regimes were compared between patients who survived and patients with a liver event (liverrelated death or transplantation) in a descriptive manner. Cirrhosis was defined by the pathologist based on liver histology. 


\section{Reference Population}

The expected number of deaths in the AIH cohort was calculated using age, gender and calendar year specific mortality risks (2006-2016) retrieved from Statistics Netherlands. For calculation of the expected death, mortality rates of 1-year age groups in 1-year periods $(1 \times 1$ format) were used, hence, ageing was taken into account. Standardized mortality ratios (SMRs), defined as the observed deaths / expected deaths, were used to compare the AIH cohort to the general Dutch population. Liver-related deaths in our cohort were compared similarly with the general population using expected number of deaths for the International Classification of Diseases-Tenth Revision codes K72-K76 and C22. These codes include deaths due to hepatic failure, cirrhosis, chronic hepatitis, other (inflammatory) liver diseases, and malignant neoplasm of liver and intrahepatic bile ducts, and do not include deaths due to alcoholic, druginduced, viral, parasitic, and other infectious liver diseases.

\section{Statistical Analysis}

Normally distributed variables were described as mean $\pm \mathrm{SD}$, and non-normally distributed variables were described as median with range or interquartile range if stated. Categorical data was described as number and percentage of total. Overall survival curves were obtained via Kaplan-Meier estimate. For differences in survival between 2 or more groups the log-rank test was used. Patients who received a liver transplantation were censored for analysis at the date of transplantation. The composite endpoint of liver-related death and transplantation was used to compare disease related events in $\mathrm{AIH}$ and its variants using Cox analysis. Ordinal and continuous variables were compared between 2 groups with the Mann Whitney test (non-normal) or 2-sided samples $t$ test (normal). For bimodal outcome variables, 2-sided Fisher's exact was used. In case continuous variables of 3 groups were compared, analysis of variance (normal distribution) or the Kruskal-Wallis (nonnormal) test was used. Post hoc analyses for correction of multiple testing were performed with the Bonferroni in case of analysis of variance and Dunn-Bonferroni in case of the Kruskal-Wallis test. The significance level $(\alpha)$ was set at .05. Statistical analysis were performed with IBM SPSS 22.0 (IBM Corp, Armonk, NY). Kaplan-Meier curves were computed with GraphPad Prism 7.02 (GraphPad Software, La Jolla, CA).

\section{Results}

Diagnostic characteristics and outcome parameters are summarized in Table 1 and included 385 patients with AIH, 29 with AIH-PBC, and 35 with AIH-PSC. The cumulative number of patient-years from diagnosis to

\section{What You Need to Know}

\section{Background}

Autoimmune hepatitis (AIH) is a chronic immunemediated inflammatory liver disease. There are few data and contradictory findings on mortality of patients with $\mathrm{AIH}$ or its variants.

\section{Findings}

Survival times of patients with AIH do not differ significantly from those of the general Dutch population unless patients also have cirrhosis or primary sclerosing cholangitis.

\section{Implications for patient care}

Patients with AIH require close monitoring to prevent cirrhosis. Survival times of patients with AIH are shortened by the liver disease itself, rather than by systemic effects of AIH or treatment-related serious adverse events.

the end of follow-up was 7217 years. All patients had a revised AIH score of $\geq 12$ (post-treatment). ${ }^{10}$ Viral hepatitis $B$ and $C$ was excluded in 446, unknown in 1 , and positive in 2 patients before AIH was diagnosed. Previously, the prevalence of hepatitis $\mathrm{E}$ was tested in a representative group of 136 patients with AIH in our cohort. No cases of acute hepatitis $\mathrm{E}$ virus infection were identified, whereas 29 patients had been exposed. ${ }^{12}$ Antinuclear antibodies and anti-smooth muscle antibodies were positive in $61 \%$ of tested patients $(n=395)$ and in $63 \%(n=379)$, respectively. In total, $86 \%$ were positive for anti-nuclear antibodies or anti-smooth muscle antibodies, in $37(8 \%)$ patients results were not available. Anti-liver/kidney microsomal antibody type 1, characteristic for AIH type 2, was positive in 17 (6\%) of 285 tested patients. In $61(14 \%)$ patients AMA or histology results were not available. Treatment details of the initial and last year of treatment are shown in Supplementary Table 1.

\section{Relapse}

A total of 200 (45\%) patients experienced 1 or more relapses. Of these patients, 25 (13\%) were weaned from therapy when this first relapse occurred, and 150 (87\%) patients relapsed while on immunosuppressive therapy.

\section{Mortality}

During the 10-year follow-up, 55 (14\%) AIH patients died at a mean age of 73 (range, 33-94) years, a median time of 142 (range, 12-471) months after diagnosis. In addition, $2(7 \%)$ patients with AIH-PBC died at the 44 and 54 years of age and 3 (9\%) AIH-PSC patients died at the 53,56 , and 57 years of age; all more than 10 years after diagnosis. 
Table 1. Characteristics at Diagnosis and Outcome Parameters

\begin{tabular}{lccc}
\hline & AlH & AlH-PBC & AlH-PSC Value \\
\hline Characteristics at diagnosis & & & $35(8)$ \\
Patients & $385(86)$ & $29(6)$ & $21(60)$ \\
Female & $300(78)$ & $27(93)$ & $30(12-54)$ \\
Age, y & $46(2-88)$ & $46(16-70)$ & $7(2-17)$ \\
MELD score & $10(0-22)$ & $8(1-22)$ & $23 \pm 96^{a}$ \\
IgG, g/L & $29 \pm 13$ & $24 \pm 13$ & $273(136-701)$ \\
ALT, U/L & $518(214-1052)$ & $274(85-623)$ & $1(3)$ \\
Cirrhosis & $52(18)$ & $4(14)$ & $29(94)$ \\
ANA and/or SMA positive & $325(91)$ & $19(70)$ & $21(70)$ \\
ANA positive & $260(76)$ & $13(52)$ & $19(63)$ \\
SMA positive & $216(66)$ & $13(54)$ & $5(0-29)$ \\
10-y follow-up & $6(0-34)$ & $5(0-8)$ & $3(9)$ \\
Time since diagnosis, y & $20(6)$ & 0 & $3(9)$ \\
Cirrhosis development & $4(1)$ & $2(7)$ & $3(9)$ \\
Liver transplantation & $55(14)$ & $2(7)$ & $3\left(107^{\circ}\right.$ \\
Deaths & $22(40.0)$ & $1(50.0)$ & $3(100.0)$ \\
Causes of death & $12(21.8)$ & &
\end{tabular}

NOTE. Values are $\mathrm{n}(\%)$, median (interquartile range), or mean $\pm \mathrm{SD}$.

$\mathrm{AlH}$, autoimmune hepatitis; AlH-PBC, autoimmune hepatitis with concurrent primary biliary cholangitis; AlH-PSC, autoimmune hepatitis with concurrent primary sclerosing cholangitis; ALT, alanine aminotransferase; ANA, anti-nuclear antibody; lgG, immunoglobulin G; MELD, Model for End-Stage Liver Disease; SMA, smooth muscle antibody.

${ }^{a}$ Post hoc analysis showed significant differences between AlH and AlH-PSC $(P=.044)$, AlH-PSC and AlH-PBC $(P=.005)$.

${ }^{b}$ Post hoc analysis showed significant differences between $\mathrm{AlH}$ and $\mathrm{AlH}-\mathrm{PSC}(P<.0001)$ and between $\mathrm{AlH}-\mathrm{PBC}$ and $\mathrm{AlH}-\mathrm{PSC}(P<.001)$.

${ }^{c}$ Post hoc analysis showed a significant $(P=.029)$ difference between $\mathrm{AlH}$ and $\mathrm{AlH}-\mathrm{PBC}$.

The overall standardized mortality rate was increased in AIH patients with cirrhosis at any time in the disease course and not in AIH patients without cirrhosis (Table 2). To prevent bias due to an overestimation of IAIHG score in the included population, we performed additional stringent analyses after the exclusion of the 61 patients who did not have histology or AMA results, as biliary changes and a positive AMA may deduct points on

Table 2. SMR

\begin{tabular}{|c|c|c|c|c|}
\hline & Observed & Expected & SMR & $95 \% \mathrm{Cl}$ \\
\hline Liver disease-related death & 26 & 0.5 & 55.2 & $37.6-81.7$ \\
\hline $\begin{array}{l}\text { Non-liver disease-related } \\
\text { death }\end{array}$ & 34 & 40.6 & 0.8 & $0.6-1.2$ \\
\hline \multicolumn{5}{|l|}{ Overall mortality } \\
\hline $\mathrm{AlH}$ & 55 & 38.8 & 1.4 & $1.1-1.8$ \\
\hline Noncirrhotic & 26 & 21.5 & 1.2 & $0.8-1.8$ \\
\hline Cirrhotic $^{a}$ & 14 & 7.3 & 1.9 & $1.2-3.4$ \\
\hline Age $\leq 50 \mathrm{y}^{b}$ & 12 & 5.6 & 2.1 & $1.3-4.0$ \\
\hline Age $>50 y^{b}$ & 43 & 33.2 & 1.3 & $0.9-1.8$ \\
\hline AlH-PBC & 2 & 1.6 & 1.2 & $0.3-4.9$ \\
\hline AlH-PSC & 3 & 0.6 & 4.7 & $1.5-14.6$ \\
\hline
\end{tabular}

$\mathrm{AlH}$, autoimmune hepatitis; AlH-PBC, autoimmune hepatitis with concurrent primary biliary cholangitis; AIH-PSC, autoimmune hepatitis with concurrent primary sclerosing cholangitis; $\mathrm{Cl}$, confidence interval; SMR, standardized mortality ratio.

${ }^{2}$ Cirrhosis at any time from diagnosis to the end of follow-up.

${ }^{b} \mathrm{Age}$ at the start of the 10 -year follow-up. the revised AIH criteria. These analyses were similar to the overall included population (Supplementary Table 2).

Kaplan Meier estimates of patients with $\mathrm{AIH}$ are shown in Figure 1. Comparison with age and gendermatched population controls shows an increased overall mortality in patients with AIH. Overall survival of AIH-PBC patients was similar $(P=.71)$ and survival of AIH-PSC patients was decreased compared with the

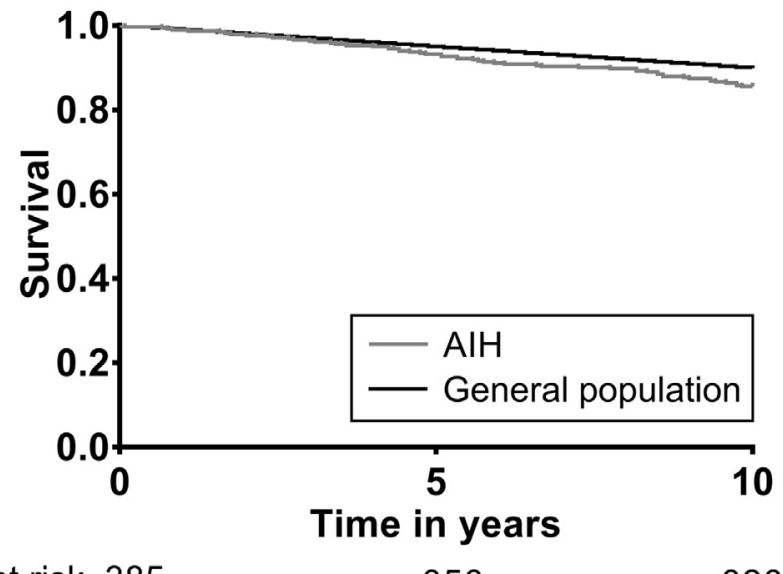

$N$ at risk 385

356 326

Figure 1. Overall survival was decreased $(P=.022)$ in patients with autoimmune hepatitis $(\mathrm{AlH})$ compared with the age- and gender-matched Dutch population. 
general Dutch population $(P=.009)$ (Supplementary Figure 1).

Survival of the composite endpoint of liver-related mortality or liver transplantation was impaired in AIH patients with cirrhosis compared patients without cirrhosis (Figure 2). Patients who reached this composite endpoint had significantly more cirrhosis, higher international normalized ratio and lower platelet and albumin levels compared with patients who survived (Table 3 ).

In uni- and multivariate Cox analysis age, cirrhosis, AIH-PSC, 4 or more relapses per decade, or not achieving remission were predictors for the composite liverrelated endpoint, whereas AIH-PBC, a pediatric disease onset, gender, or $<4$ relapses per decade were not (Table 4). Additional analysis of liver-related deaths in AIH patients without censoring liver transplantations shows that patients who died of liver-related events had histological proven cirrhosis more often compared with patients who survived (41\% vs $19 \% ; P=.01$ ). In this Cox regression analysis, histological proven cirrhosis $(P<.001)$, corrected for age at baseline, was a predicting factor $(P=.001)$ for liver-related death.

Supplementary Figure 2 shows survival of the composite liver-related endpoint for patients with $\mathrm{AIH}, \mathrm{AIH}-$ $\mathrm{PBC}$, and AIH-PSC.

Twenty-three patients developed cirrhosis during therapy, of whom 15 had fibrosis (stage 1-3) at diagnosis. In all cases, cirrhosis was histologically confirmed before August 1, 2006. Both patients who had cirrhosis at diagnosis (hazard ratio, $2.5 ; 95 \%$ confidence interval, 1.0-6.2; $P=.05$ ) and patients who developed cirrhosis after diagnosis (hazard ratio, 4.6; 95\% confidence interval, 1.5-14.0; $P<.01$ ) had an increased risk of liverrelated death or liver transplantation, when compared with patients without cirrhosis.

The cause of death was established in 58 patients (Table 1). Hepatobiliary disease accounted for 26 deaths,

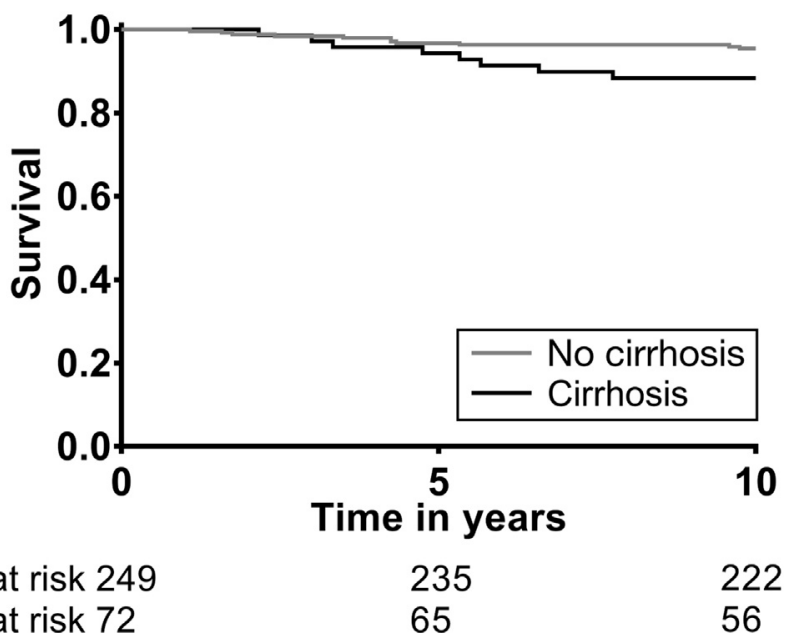

Table 3. Patient Characteristics Stratified by Survival of Liver-Related Event

\begin{tabular}{|c|c|c|c|}
\hline & Survival & $\begin{array}{l}\text { Liver-Related } \\
\text { Death or Liver } \\
\text { Transplantation }\end{array}$ & $\begin{array}{c}P \\
\text { Value }\end{array}$ \\
\hline Patients & $414(92)$ & $35(8)$ & \\
\hline Female, \% & 78 & 71 & .399 \\
\hline Cirrhosis anytime & $68(16)$ & $11(31)$ & .013 \\
\hline Relapse + LOR & $2(0-4)$ & $2(0-4)$ & .876 \\
\hline Remission achieved & $375(91)$ & $29(83)$ & .126 \\
\hline Time to remission, mo & $4(0-167)$ & $3(1-35)$ & .825 \\
\hline $\begin{array}{l}\text { Second- or third-line } \\
\text { therapy }\end{array}$ & $42(10)$ & $13(37)$ & $<.001$ \\
\hline First-line treatment & $387(95)$ & $34(97)$ & .643 \\
\hline $\begin{array}{l}\text { Prednisone and } \\
\text { azathioprine }\end{array}$ & $292(72)$ & $26(77)$ & - \\
\hline Prednisone monotherapy & $62(15)$ & $6(18)$ & - \\
\hline $\begin{array}{l}\text { Azathioprine monotherapy } \\
\text { At the time of diagnosis }\end{array}$ & $33(8)$ & $1(3)$ & - \\
\hline Age, y & $43(2-88)$ & $47(10-81)$ & .183 \\
\hline MELD score & $10(0-32)$ & $11(5-22)$ & .199 \\
\hline Creatinine, $\mu \mathrm{mol} / \mathrm{L}$ & $73(40-158)$ & $66(47-98)$ & .195 \\
\hline Bilirubin, $\mu \mathrm{mol} / \mathrm{L}$ & 38 (4-719) & $48(9-353)$ & .368 \\
\hline INR & $1.1(0.8-4.7)$ & $1.3(1.0-4.1)$ & .006 \\
\hline Albumin, $g / L$ & $38(17-54)$ & $35(26-44)$ & .013 \\
\hline Platelets $\left(\times 10^{9} / \mathrm{L}\right)$ & $210(61-634)$ & $142(63-232)$ & $<.001$ \\
\hline $\mathrm{ALT}, \mathrm{U} / \mathrm{L}$ & $484(13-4693)$ & $228(36-3080)$ & .259 \\
\hline $\operatorname{lgG}, \mathrm{g} / \mathrm{L}$ & $26(7-91)$ & $23(9-51)$ & .860 \\
\hline
\end{tabular}

NOTE: Values are $\mathrm{n}(\%)$ or median (range), unless otherwise indicated.

ALT, alanine aminotransferase; IgG, immunoglobulin G; INR, international normalized ratio; LOR, loss of remission; MELD, Model for End-Stage Liver Disease.

resulting in a marked increase of liver-related mortality in AIH (SMR, 50.0; 95\% confidence interval, 33.0-76.0), AIH-PBC (SMR, 48.3; 95\% confidence interval, 6.8-343.2), and AIH-PSC (SMR, 278.3; 95\% confidence

Table 4. Survival of Liver-Related Death and Liver Transplantation

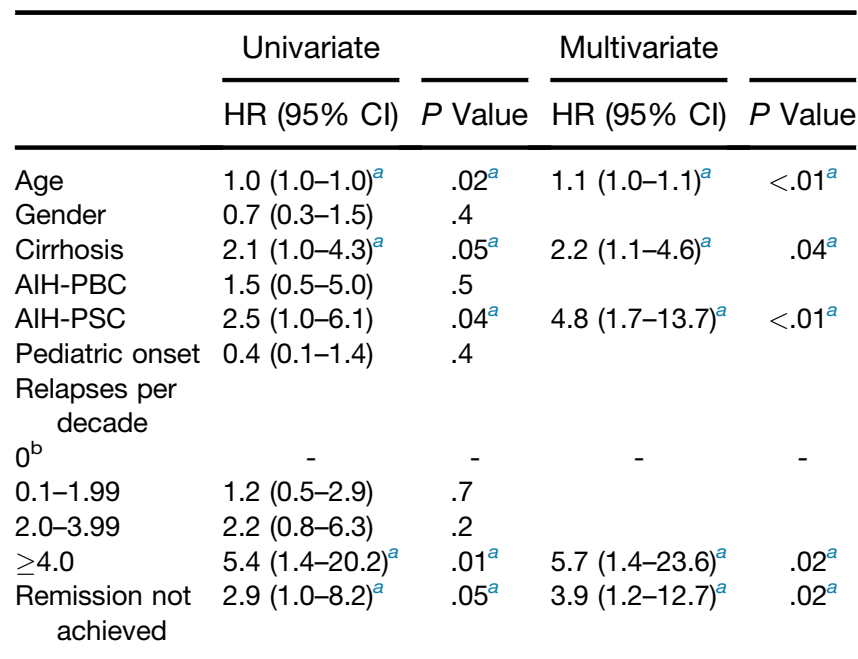

$\mathrm{AlH}$, autoimmune hepatitis; $\mathrm{AlH}-\mathrm{PBC}$, autoimmune hepatitis with concurrent primary biliary cholangitis; AlH-PSC, autoimmune hepatitis with concurrent primary sclerosing cholangitis; $\mathrm{Cl}$, confidence interval; $\mathrm{HR}$, hazard ratio. ${ }^{a}$ Reference category. 
interval, 89.8-863.0) compared with the general Dutch population. Patients who died due to hepatobiliary disease encountered multiple $(n=47)$ complications contributing to death (Supplementary Table 3). The 3 patients who died due to hepatocellular carcinoma had cirrhosis. The estimated incidence of mortality due to HCC in patients with cirrhosis at diagnosis in our study was 3.6 per 1000 patient-years at risk. In 2 patients, the cause of death could not be unraveled. Survival of patients who deceased due to non-liver-related causes was not different from the general population, indicating that excess mortality is driven by the underlying liver disease (Table 2).

Death likely related to immunosuppressive therapy occurred in one 44-year-old patient who died due to a pulmonary aspergillus infection while being treated with budesonide, ciclosporin, and mycophenolate mofetil. Mortality due to extrahepatic malignancies included melanoma $(\mathrm{n}=1)$, lung cancer $(\mathrm{n}=2)$, prostate cancer $(\mathrm{n}=1)$, colorectal cancer $(\mathrm{n}=3)$, vulvar cancer $(\mathrm{n}=1)$, urothelial cancer $(\mathrm{n}=1)$, ovarian cancer $(\mathrm{n}=1)$, and 2 metastatic malignancies with unknown primary tumors.

Patients who died due to hepatobiliary causes deceased at a significantly younger age than did those who died of other causes (median age 68 vs 80 years, $P=.013$ ). Subgroup analysis for age at start of follow-up revealed that patients 50 years of age or younger had a higher SMR than patients older than 50 years of age, compared with the all-cause mortality of the general population (Table 2). This could be explained by the low number of deaths in the general population 50 years of age or younger.

Survival rates of patients who were managed in academic hospitals and nonacademic hospitals were comparable ( $87 \%$ vs $84 \%$; $P=.27$ ).

\section{Liver Transplantation}

During the 10-year follow-up, 9 (2\%) patients were transplanted at a median age of 48 (range, 27-67) years, of whom 4 were AIH patients (1\%), 2 were AIH-PBC patients (7\%) and 3 were AIH-PSC patients (9\%). Seven transplanted patients were alive at the end of follow-up, 2 died of extrahepatic malignancies (lung cancer and melanoma) and were censored for mortality analysis at the date of transplantation. In addition, $4 \mathrm{AIH}$, 2 AIH-PBC, and 3 AIH-PSC patients were positively screened for, but did not receive, a liver transplantation. Two AIH, 1 AIH-PBC, and 1 AIH-PSC patient died of liverrelated disease while waiting for a donor liver.

\section{Discussion}

Published data on mortality rates in AIH patients and its variants are scarce and contradictory. In some small and mostly single center studies the observed mortality rates of AIH patients was comparable to the general population. ${ }^{3-5}$ Other larger studies showed an increased mortality in AIH patients SMR. ${ }^{3-6,13-18}$ As far as we know the current study comprises the largest cohort of patients with established $\mathrm{AIH}$ and a follow-up of $>10$ years. Patients were recruited from general hospitals, tertiary referral centers, and transplantation hospitals and thus reflects a realistic representation of "real world" AIH. The findings of our study are reassuring. Patients without cirrhosis have a normal life expectancy. Only patients with cirrhosis and particularly AIH-PSC patients have an impaired survival, which can be entirely attributed to liver-related complications. No excess mortality was seen in deaths unrelated to the underlying liver disease which is in line with findings of Hoeroldt et $\mathrm{al}^{6}{ }^{6}$ suggesting that the liver disease and no other factors, including therapy, are responsible for the excess mortality.

We found that liver disease was responsible for approximately one third of the deaths in AIH patients, consistent with $39 \%-55 \%$ of deaths accounted to liver disease in other studies. ${ }^{7,15}$ These studies did not report the number of patients with variant syndromes, which generally constitutes a proportion of $10 \%-15 \%$ of $\mathrm{AIH}$ patients. ${ }^{19}$ In this study, we addressed differences in outcomes among AIH, AIH-PBC, and AIH-PSC. We found that increased overall mortality rate was most profound in the relatively young group of AIH-PSC patients, which was entirely attributable to the underlying liver disease. No excess mortality was seen for AIH-PBC patients. Although the latter finding may be explained by the small number of patients in this group, it is consistent with other reports in the literature. ${ }^{20}$ AIH-PBC was defined as AIH with features of PBC and compatible histology, which is less stringent than the Paris criteria; however, we were not able to perform a centralized review of biopsies. The incidence of mortality due to HCC in patients with cirrhosis at diagnosis was markedly lower in our study than the incidence of HCC in found by Tansel et al. ${ }^{21}$ This may in part be explained by the difference in study design. Furthermore, we cannot exclude that the HCC incidence is underestimated because of missing cases, as cross-referencing with the Dutch cancer registry was not possible.

In our cohort of relatively young patients, $8 \%$ of the patients reached the composite liver disease endpoint of liver-related death or liver transplantation over a 10year period. Cirrhosis, age, AIH-PSC, more than 4 relapses per decade, and not achieving remission were prognostic for this composite liver-related outcome in uni- and multivariate Cox analysis, which is in line with other studies. ${ }^{6,22}$ Prior studies have shown that pediatric onset patients have a more aggressive disease course, yet we did not observe a correlation between a pediatric onset and liver-related endpoints, which is likely due to the relatively low number of pediatric onset patients in our cohort. ${ }^{23,24}$

Patients who reached the composite liver-related outcome had lower platelet counts, lower albumin 
levels, an increased international normalized ratio, and cirrhosis more frequently, compared with patients who survived. The differences in laboratory values are likely a result of cirrhosis; however, this could not be confirmed in our study in an independent multivariate analysis, because of incomplete data capture on the laboratory parameters. In support of this figure, cirrhosis was identified using albumin, international normalized ratio, and the platelet count in a meta-analysis of patients with liver disease. ${ }^{25}$

Survival of patients with cirrhosis in our cohort was, again, reduced when liver-related deaths without liver transplantations were analyzed, furthermore confirming the prognostic value of cirrhosis. Hence, even when transplantation is successful, cirrhosis is still prognostic for liver-related death. Both patients with cirrhosis at diagnosis and patients who developed cirrhosis during the disease course had an impaired survival of the composite liver-related endpoint, compared with patients without cirrhosis. Not surprisingly, second- or third-line therapy were more frequently required during the disease course of patients who eventually reached the composite liver-related endpoint. Malignancies have been reported in relation to long-term immunosuppressive therapy. ${ }^{26}$ In our cohort no excess of non-liverrelated mortality including mortality due to malignancies was noticed, although 1 patient died of causes related to immunosuppressive therapy. The decreased survival was driven by the underlying liver disease and not by systemic effects of AIH or treatment related serious adverse events. The number of patients subjected to liver transplantation was low (2\%) in our cohort compared with reported numbers of $10 \%{ }^{27}$ and $5 \%{ }^{15}$ reported in 2006 and 2010, respectively. In our cohort another 9 (2\%) patients were listed for liver transplantation, of whom 4 died without being transplanted. Patients with variant syndromes were more likely to be placed on the transplantation list than AIH patients.

The strengths of this study are defined by the largest number of included patients with established AIH in both transplant, nontransplant and general referral hospitals, and the certainty that no patients who reached an endpoint were missed. In addition, the design of this cohort study, following onward those AIH patients who were alive in 2006, allowed for a SMR assessment compared with the general Dutch population. However, those patients who had reached an endpoint before baseline were excluded due to this study design. This fact and the expected boundaries of a retrospective study, such as missing data, precluding large scale multivariate regression analysis, are limitations that should be noted. We aimed to address these issues by performing a stringent analysis approach in those patients with missing data in histology and AMA status.

In conclusion, patients with AIH without cirrhosis have a similar survival rate as the general population. Patients with AIH and cirrhosis and patients with an $\mathrm{AIH}-$ PSC variant syndrome have a significant increased risk of death and liver transplantation. This impaired survival rate can entirely be attributed to liver-related complications.

\section{Supplementary Material}

Note: To access the supplementary material accompanying this article, visit the online version of Clinical Gastroenterology and Hepatology at www.cghjournal.org, and at https://doi.org/10.1016/j.cgh.2018.09.046.

\section{References}

1. Manns MP, Czaja AJ, Gorham JD, et al. Diagnosis and management of autoimmune hepatitis. Hepatology 2010; 51:2193-2213.

2. Lamers MM, van Oijen MG, Pronk M, et al. Treatment options for autoimmune hepatitis: a systematic review of randomized controlled trials. J Hepatol 2010;53:191-108.

3. Yoshizawa K, Matsumoto A, Ichijo T, et al. Long-term outcome of Japanese patients with type 1 autoimmune hepatitis. Hepatology 2012;56:668-676.

4. Kanzler S, Lohr H, Gerken G, et al. Long-term management and prognosis of autoimmune hepatitis (AlH): a single center experience. Z Gastroenterol 2001;39:339-341, 344-348.

5. Malekzadeh Z, Haghazali S, Sepanlou SG, et al. Clinical features and long term outcome of 102 treated autoimmune hepatitis patients. Hepat Mon 2012;12:92-99.

6. Hoeroldt B, McFarlane E, Dube A, et al. Long-term outcomes of patients with autoimmune hepatitis managed at a nontransplant center. Gastroenterology 2011;140:1980-1989.

7. Gronbaek L, Vilstrup H, Jepsen P. Autoimmune hepatitis in Denmark: incidence, prevalence, prognosis, and causes of death. A nationwide registry-based cohort study. J Hepatol 2014;60:612-617.

8. Yang F, Wang Q, Wang Z, et al. The natural history and prognosis of primary biliary cirrhosis with clinical features of autoimmune hepatitis. Clin Rev Allergy Immunol 2016; 50:114-123.

9. van Gerven NM, Verwer BJ, Witte BI, et al. Epidemiology and clinical characteristics of autoimmune hepatitis in the Netherlands. Scand J Gastroenterol 2014;49:1245-1254.

10. Alvarez F, Berg PA, Bianchi FB, et al. International Autoimmune Hepatitis Group Report: review of criteria for diagnosis of autoimmune hepatitis. J Hepatol 1999;31:929-938.

11. van Gerven NM, Verwer BJ, Witte BI, et al. Relapse is almost universal after withdrawal of immunosuppressive medication in patients with autoimmune hepatitis in remission. J Hepatol 2013;58:141-147.

12. van Gerven NM, van der Eijk AA, Pas SD, et al. Seroprevalence of hepatitis $E$ virus in autoimmune hepatitis patients in the Netherlands. J Gastrointestin Liver Dis 2016;25:9-13.

13. Dhaliwal HK, Hoeroldt BS, Dube AK, et al. Long-term prognostic significance of persisting histological activity despite biochemical remission in autoimmune hepatitis. Am J Gastroenterol 2015;110:993-999.

14. Ngu JH, Gearry RB, Frampton CM, et al. Mortality and the risk of malignancy in autoimmune liver diseases: a population-based study in Canterbury, New Zealand. Hepatology 2012; 55:522-529. 
15. Werner M, Wallerstedt S, Lindgren S, et al. Characteristics and long-term outcome of patients with autoimmune hepatitis related to the initial treatment response. Scand J Gastroenterol 2010;45:457-467.

16. Feld JJ, Dinh H, Arenovich T, et al. Autoimmune hepatitis: effect of symptoms and cirrhosis on natural history and outcome. Hepatology 2005;42:53-62.

17. Al-Chalabi T, Underhill JA, Portmann BC, et al. Impact of gender on the long-term outcome and survival of patients with autoimmune hepatitis. J Hepatol 2008;48:140-147.

18. Roberts SK, Therneau TM, Czaja AJ. Prognosis of histological cirrhosis in type 1 autoimmune hepatitis. Gastroenterology 1996;110:848-857.

19. Boberg KM, Chapman RW, Hirschfield GM, et al. Overlap syndromes: the International Autoimmune Hepatitis Group (IAIHG) position statement on a controversial issue. J Hepatol 2011; 54:374-385.

20. Al-Chalabi T, Portmann BC, Bernal W, et al. Autoimmune hepatitis overlap syndromes: an evaluation of treatment response, long-term outcome and survival. Aliment Pharmacol Ther 2008; 28:209-220.

21. Tansel A, Katz LH, El-Serag HB, et al. Incidence and determinants of hepatocellular carcinoma in autoimmune hepatitis: a systematic review and meta-analysis. Clin Gastroenterol Hepatol 2017;15:1207-1217.e4.

22. Montano-Loza AJ, Carpenter HA, Czaja AJ. Consequences of treatment withdrawal in type 1 autoimmune hepatitis. Liver Int 2007;27:507-515.
23. Gregorio GV, Portmann B, Reid F, et al. Autoimmune hepatitis in childhood: a 20-year experience. Hepatology 1997;25:541-547.

24. Maggiore G, Bernard O, Hadchouel M, et al. Treatment of autoimmune chronic active hepatitis in childhood. J Pediatr 1984;104:839-844.

25. Udell JA, Wang CS, Tinmouth J, et al. Does this patient with liver disease have cirrhosis? JAMA 2012;307:832-842.

26. Farraye FA, Melmed GY, Lichtenstein GR, et al. ACG clinical guideline: preventive care in inflammatory bowel disease. Am J Gastroenterol 2017;112:241-258.

27. Manns MP, Vogel A. Autoimmune hepatitis, from mechanisms to therapy. Hepatology 2006;43:S132-S144.

\section{Reprint requests}

Address requests for reprints to: Gerd Bouma, Amsterdam UMC, VUmc, Department of Gastroenterology and Hepatology, Amsterdam Gastroenterology and Metabolism, de Boelelaan 1117, $1081 \mathrm{HV}$, Amsterdam, the Netherlands. e-mail: g.bouma@vumc.nl; fax: +3120-444 0554.

\section{Acknowledgments}

Dutch Autoimmune Hepatitis Study Group collaborators: T.C.M.A. Schreuder (University Medical Center Groningen), E.J. van der Wouden (Isala Hospital, Zwolle), J.J.M. van Meyel and L.C. Baak (OLVG, Amsterdam), P.H.G.M. Stadhouders P.H.G.M. Stadhouders (St. Antonius Hospital, Nieuwegein), M. Klemt-Kropp (Noordwest Ziekenhuisgroep, Alkmaar), M.A.M.T. Verhagen (Diakonessenhuis, Utrecht), A. Bhalla (HagaZiekenhuis, Den Haag), J.Ph. Kuijvenhoven (Spaarne Gasthuis, Haarlem).

\section{Conflicts of interest}

The authors disclose no conflicts. 

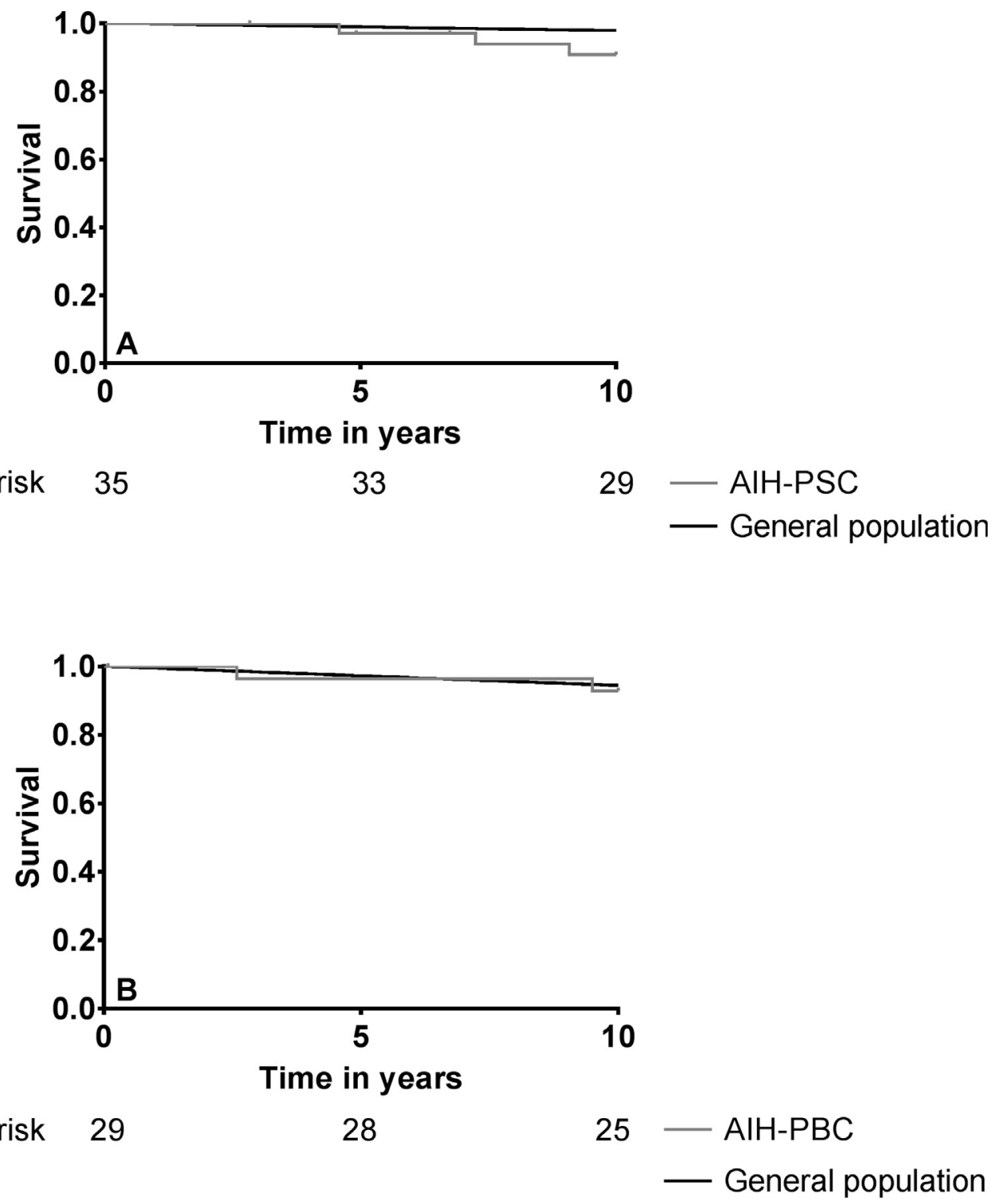

Supplementary Figure 1. Kaplan-Meier estimates of overall survival. $(A)$ Survival of patients with autoimmune hepatitis with primary sclerosing cholangitis (AlH-PSC) was decreased compared with the age- and gender-matched population $(P=.009)$ $(B)$ Cumulative survival of AlH with concurrent primary biliary cholangitis (AlH-PBC) did not differ from the age- and gendermatched population $(0.714)$

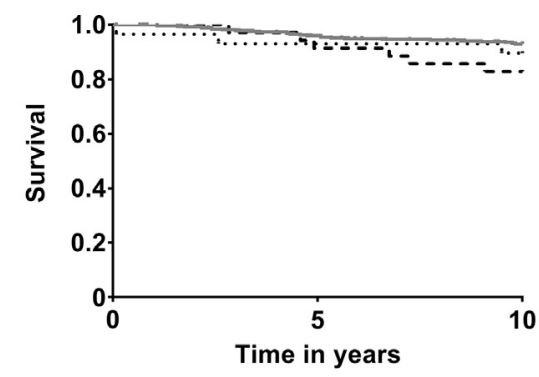

$\begin{array}{lllll}N \text { at risk } & 385 & 355 & 326 & - \text { AlH } \\ N \text { at risk } & 29 & 28 & 25 & \ldots \text { AlH-PBC } \\ N \text { at risk } & 35 & 33 & 29 & -\cdots \text { AlH-PSC }\end{array}$

Supplementary Figure 2. Survival functions of patients with autoimmune hepatitis $(\mathrm{AlH})$, AlH with concurrent primary biliary cholangitis (AlH-PBC) and $\mathrm{AlH}$ with primary sclerosing cholangitis (AlH-PSC) for the composite endpoint of liverrelated death or liver transplantation $(P=.081)$. 
Supplementary Table 1. Treatment Details of in the First and Last Year of Treatment

\begin{tabular}{|c|c|c|c|c|}
\hline & \multicolumn{2}{|c|}{ Initial Year of Treatment } & \multicolumn{2}{|c|}{ Last Year of Treatment } \\
\hline & Patients & Dose $(\mathrm{mg} / \mathrm{d})$ & Patients & Dose (mg/d) \\
\hline Budesonide & $4(1.1)$ & $8(3-9)$ & $45(11)$ & $6(1-9)$ \\
\hline Azathioprine & $233(66)$ & $50(4-200)$ & $256(62)$ & 75 (13-200) \\
\hline \multicolumn{5}{|l|}{ Second line } \\
\hline Tacrolimus & - & - & $5(1.2)$ & $5(1-7)$ \\
\hline Mercaptopurine & - & - & $7(1.7)$ & $50(25-75)$ \\
\hline Thioguanine & $1(0.3)$ & 16 & $3(0.7)$ & $21(3-50)$ \\
\hline
\end{tabular}

NOTE. Values are $\mathrm{n}(\%)$ or median (interquartile range). The mean time interval between initial and last year of treatment was $16 \pm 7$ years.

Supplementary Table 2. Stringent SMR Analysis

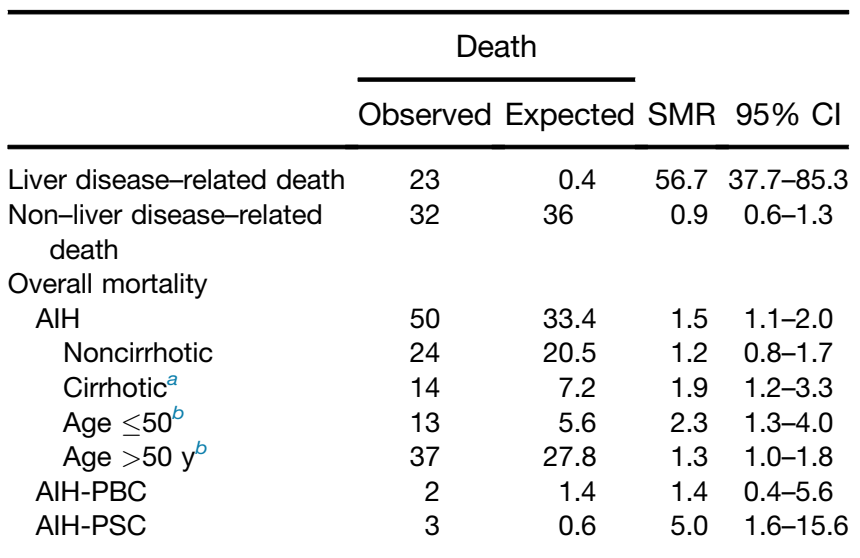

NOTE. AlH, autoimmune hepatitis; AlH-PBC, autoimmune hepatitis with concurrent primary biliary cholangitis; AIH-PSC, autoimmune hepatitis with concurrent primary sclerosing cholangitis; $\mathrm{Cl}$, confidence interval; SMR, standardized mortality ratio.

${ }^{a}$ Cirrhosis at any time from diagnosis to the end of follow-up.

${ }^{b} \mathrm{Age}$ at the start of the 10-year follow-up.
Supplementary Table 3. Complications Contributing to Death in Patients Who Died of Hepatobiliary Diseases

\begin{tabular}{lccc}
\hline & $\begin{array}{c}\text { AlH } \\
\mathrm{n}=22\end{array}$ & $\begin{array}{c}\text { AlH-PBC } \\
\mathrm{n}=1\end{array}$ & $\begin{array}{c}\text { AlH-PSC } \\
\mathrm{n}=3\end{array}$ \\
\hline $\begin{array}{l}\text { Decompensated cirrhosis or liver } \\
\text { insufficiency }\end{array}$ & 17 & 1 & 2 \\
$\begin{array}{l}\text { Gastrointestinal hemorrhage } \\
\text { Hepatocellular carcinoma }\end{array}$ & 6 & & 1 \\
$\begin{array}{l}\text { Cholangiocarcinoma } \\
\text { Hepatorenal syndrome }\end{array}$ & 2 & & 1 \\
$\begin{array}{l}\text { Spontaneous bacterial } \\
\quad \text { peritonitis }\end{array}$ & 4 & 1 & 1 \\
$\begin{array}{l}\text { Hepatic encephalopathy } \\
\text { Respiratory insufficiency }\end{array}$ & 3 & & \\
Sepsis & 3 & & \\
Severe coagulopathy & 2 & & \\
& 1 & & \\
\hline
\end{tabular}

NOTE. Some patients encountered more than 1 complication.

$\mathrm{AlH}$, autoimmune hepatitis; AlH-PBC, autoimmune hepatitis with concurrent primary biliary cholangitis; AlH-PSC, autoimmune hepatitis with concurrent primary sclerosing cholangitis.

${ }^{a}$ One patient died due to Aspergillus pneumoniae and another patient had severe dyspnea subsequent to liver failure. 